\title{
含苯胺基硫胒单元的咔唑衍生物的合成及其对阴离子的识别传感研究
}

\author{
塞军友问柏任潘顶伍谭赞 \\ 吕新阳杜欢鲍小平* \\ (贵州大学精细化工研究开发中心 绿色农药与农业生物工程国家重点实验室培育基地 \\ 教育部绿色农药与农业生物工程重点实验室 贵阳 550025)
}

\begin{abstract}
摘要 以咔唑为起始原料设计合成了含苯胺基硫脲识别单元的咔唑类阴离子受体 $\mathbf{L}$ ，通过 ${ }^{1} \mathrm{H} N M R 、{ }^{13} \mathrm{C} N \mathrm{NM} 、 \mathrm{MS}$ 、 IR 和元素分析对其结构进行了表征. UV-Vis 和荧光滴定实验表明，在二甲基亚砜(DMSO)中 $\mathbf{L}$ 能较好地识别 $\mathrm{F}^{-}$、AcO $\mathrm{Ac}^{-}$ 和 $\mathrm{H}_{2} \mathrm{PO}_{4}^{-}$且对它们表现出强络合作用 $\left(K_{\mathrm{a}}>10^{4} \mathrm{~L} / \mathrm{mol}\right)$, 识别过程伴随着肉眼可见的溶液颜色变化和明显的苂光猝灭, 这表明了受体 $\mathbf{L}$ 可作为阴离子的比色和荧光传感器. 有趣的是, 在含水 $10 \%$ 的 $\mathrm{DMSO}$ 中 $\mathbf{L}$ 能选择性识别 $\mathrm{F}^{-}$和 $\mathrm{AcO}^{-}$; 在含水 $20 \%$ 的 DMSO 中 $\mathbf{L}$ 实现了对 $\mathrm{AcO}^{-}$的专一性识别.
\end{abstract}

关键词＼cjkstart苯胺基硫脲；咔唑；氢键；阴离子识别；传感

\section{Synthesis, Recognition and Sensing Properties for Anions Based on a Carbazole Derivative Containing Phenylaminothiourea Units}

\author{
Jian, Junyou Yan, Boren Pan, Dingwu Tan, Zan \\ Lü, Xinyang Du, Huan Bao, Xiaoping*
}

(Center for Research and Development of Fine Chemicals, State Key Laboratory Breeding Base of Green Pesticide and Agricultural Bioengineering, Key Laboratory of Green Pesticide and Agricultural Bioengineering, Ministry of Education, Guizhou University, Guiyang 550025)

\begin{abstract}
A carbazole-based anion receptor (L) containing phenylaminothiourea recognition units was designed and synthesized using carbazole as starting compound, and its structure was characterized by ${ }^{1} \mathrm{H} N M R,{ }^{13} \mathrm{C}$ NMR, MS, IR and elemental analysis. UV-Vis and fluorescence titration experiments indicated that $\mathbf{L}$ selectively recognized $\mathrm{F}^{-}$, $\mathrm{AcO}^{-}$and $\mathrm{H}_{2} \mathrm{PO}_{4}^{-}$in DMSO, showing strong interactions $\left(K_{\mathrm{a}}>10^{4} \mathrm{~L} / \mathrm{mol}\right)$ with these three anions. During the anion recognition process, a naked-eye detectable color change and a distinct fluorescence quenching of $\mathbf{L}$ were simultaneously observed, indicative of its potential as colorimetric and fluorescent sensors for anions. Interestingly, only $\mathrm{F}^{-}$and $\mathrm{AcO}^{-}$could be recognized by $\mathbf{L}$ in DMSO containing $10 \%(V / V) \mathrm{H}_{2} \mathrm{O}$; a specific recognition of $\mathbf{L}$ towards $\mathrm{AcO}^{-}$was realized in DMSO containing $20 \%(V / V)$ $\mathrm{H}_{2} \mathrm{O}$.

Keywords phenylaminothiourea; carbazole; hydrogen bond; anion recognition; sensing
\end{abstract}

鉴于阴离子在化学、生物和环境等领域所起的重要 作用, 设计合成具有选择性识别和传感能力的阴离子受 体已成为当前超分子化学的一个研究热点 ${ }^{[1,2]}$. 其中, 能 将微观的阴离子识别事件转化为宏观易测的溶液颜色 或荧光变化的阴离子受体(即阴离子比色/荧光传感器) 在检测中具有仪器廉价、操作简单和容易监控等优点, 因而受到了人们的特别关注 ${ }^{[3,4]}$. 氢键型阴离子受体是
目前研究最为广泛的一类阴离子受体, 常见的氢键供体 有(硫)脲、酰胺、磺胺、吡咯、吲哚和羟基等单元. 与 其他氢键供体相比, 氨基硫腿基团能提供更多的 $\mathrm{NH}$ 位 点参与对阴离子的氢键络合，但基于该基团的阴离子受 体目前报道还很少 ${ }^{[5 \sim 10]}$.

咔唑类化合物易于进行化学修饰, 具有特殊刚性结 构和较大共轭体系, 其分子可发蓝色苂光; 更重要的是,

\footnotetext{
*E-mail: baoxp_1980@aliyun.com

Received October 22, 2014; revised December 10, 2014; published online January 6, 2015.

Project supported by the National Natural Science Foundation of China (No. 21161005).

国家自然科学基金(No. 21161005)资助项目.
} 
咔唑 9 位 NH 本身即可作氢键供体单元使用，这些优势 使咔唑类阴离子受体化学近年来得以快速发展 ${ }^{[11]}$. 最 近我们课题组 ${ }^{[12]}$ 报道了含磺酰肼单元的咔唑类阴离子 受体对阴离子的识别性能, 测试表明该受体在 DMSO 中能选择性地识别 $\mathrm{F}^{-} 、 \mathrm{AcO}^{-}$和 $\mathrm{H}_{2} \mathrm{PO}_{4}^{-}$, 但对它们只表 现出中等强度的亲和性 $\left(\approx 10^{3} \mathrm{~L} / \mathrm{mol}\right)$. 文献检索表明, 将氨基硫嫝单元引入到咔唑分子骨架中来合成具有光 学响应的阴离子受体目前还未见报道. 本文以咔唑为起 始原料经五步反应合成了含苯胺基硫脲单元为识别位 点的阴离子受体 $\mathbf{L}$, 合成路线见 Scheme 1. L 中咔唑 9 位 $\mathrm{NH}$ 和 6 个苯胺基硫脲 $\mathrm{NH}$ 可协同起来键联阴离子, 咔唑骨架自身充当信号单元可提供识别过程中的 $U V-V$ is 和苂光响应. 利用 UV-Vis、苂光和核磁滴定等 方法研究了 $\mathbf{L}$ 在二甲基亚砜(DMSO)及含水 DMSO 中对 不同阴离子的识别传感作用, 并提出了 $\mathbf{L}$ 与 $\mathrm{F}^{-}$的可能 结合模式.

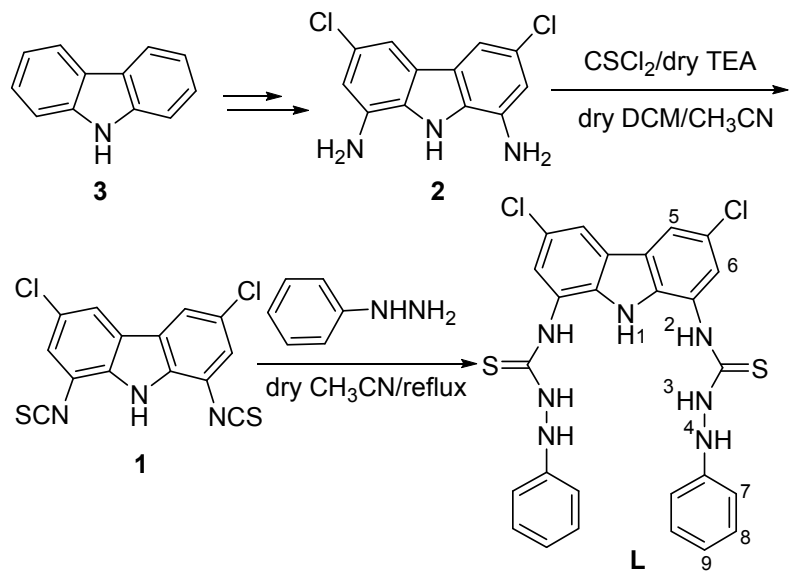

图式 1 受体 $\mathbf{L}$ 的合成路线

Scheme 1 Synthesis of receptor $\mathbf{L}$

\section{1 结果与讨论}

\subsection{UV-Vis 光谱滴定}

图 1 为在受体 $\mathbf{L}$ 的 DMSO 溶液中加入 4.0 equiv. 不 同阴离子后的吸收光谱变化. 由图 1 可知, 只有 $\mathrm{F}^{-}$、 $\mathrm{AcO}^{-}$和 $\mathrm{H}_{2} \mathrm{PO}_{4}^{-}$造成 $\mathbf{L}$ 的吸收光谱发生了明显变化, 其他阴离子 $\left(\mathrm{HSO}_{4}^{-} 、 \mathrm{Cl}^{-} 、 \mathrm{NO}_{3}^{-} 、 \mathrm{~N}_{3}^{-} 、 \mathrm{ClO}_{4}^{-} 、 \mathrm{Br}^{-}\right.$ 和 $\mathrm{I}^{-}$) 对 $\mathbf{L}$ 的吸收光谱无影响. 值得注意的是, $\mathrm{L}$ 与上述 3 种离子作用后其溶液由无色变为淡黄色 $(300 \sim 450 \mathrm{~nm}$ 吸收带显著增强所致), 这实现了对这些阴离子的裸眼 检测(图 2). 如图 3 所示, 随着加入的 $\mathrm{F}^{-}$浓度不断增加, $\mathbf{L}$ 在 $258 \mathrm{~nm}$ 处的吸收峰及在 $350 \mathrm{~nm}$ 处的肩峰逐渐增强并 伴有一定的红移, 其中以 $328 \mathrm{~nm}$ 为中心的咔唑骨架吸 收带增强最为明显. Job 曲线法(图 4)及良好的 $1: 1$ 非线 性拟合结果(图 3 插图)均证明受体 $\mathbf{L}$ 与 $\mathrm{F}^{-}$在 DMSO 中 形成了化学计量比为 $1: 1$ 的配合物. 由图 5 可知, $\mathbf{L}$ 对
$\mathrm{F}^{-}$的检测限为 $6.81 \mu \mathrm{mol} / \mathrm{L}^{[13]}$. 向 $\mathbf{L}$ 的 DMSO 溶液中加 入 $\mathrm{AcO}^{-}$或 $\mathrm{H}_{2} \mathrm{PO}_{4}^{-}$后, 其吸收光谱变化与 $\mathrm{F}^{-}$相似, $\mathbf{L}$ 对这两种离子的检测限分别为 4.58 和 $4.80 \mu \mathrm{mol} / \mathrm{L}$.

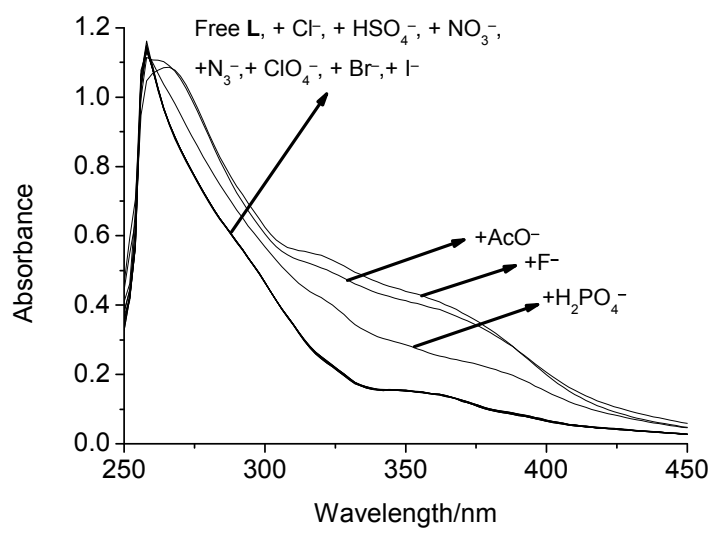

图 1 受体 $\mathbf{L}(20 \mu \mathrm{mol} / \mathrm{L})$ 在 $\mathrm{DMSO}$ 中与 4.0 equiv. 不同阴离子 作用后的 UV-Vis 变化图

Figure 1 UV-Vis spectral changes of DMSO solution of receptor $\mathbf{L}(20 \mu \mathrm{mol} / \mathrm{L})$ upon addition of 4.0 equiv. of different anions

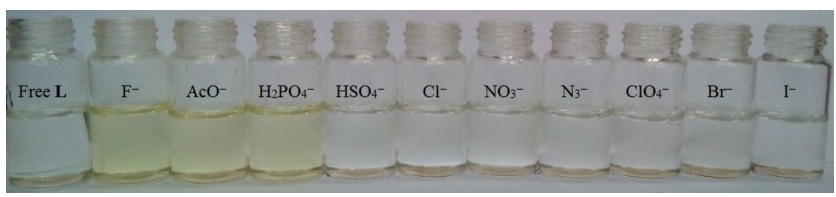

图 2 受体 $\mathbf{L}(20 \mu \mathrm{mol} / \mathrm{L})$ 的 $\mathrm{DMSO}$ 溶液加入 5.0 equiv. 不同阴 离子后的颜色变化

Figure 2 Color changes of DMSO solution of receptor $\mathbf{L}$ (20 $\mu \mathrm{mol} / \mathrm{L}$ ) upon addition of 5.0 equiv. different anions

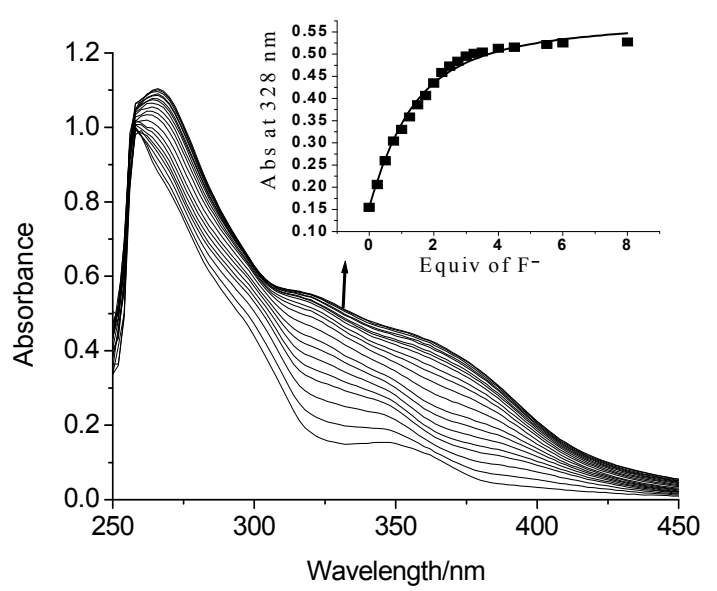

图 3 受体 $\mathbf{L}(20 \mu \mathrm{mol} / \mathrm{L})$ 在 $\mathrm{DMSO}$ 中与 $\mathrm{F}^{-}$作用后的 UV-Vis 光谱变化

Figure 3 UV-Vis spectral changes of receptor $\mathbf{L}(20 \mu \mathrm{mol} / \mathrm{L})$ in DMSO upon addition of $\mathrm{F}^{-}$

The inset was the nonlinear curve fitting of the absorbance at $328 \mathrm{~nm}$ against added $\mathrm{F}^{-}$using a $1: 1$ binding model

为探求受体 $\mathbf{L}$ 的实际用途并进一步改善其识别选 择性, 我们又在含水 DMSO 中考察了 $\mathbf{L}$ 对阴离子的识别 


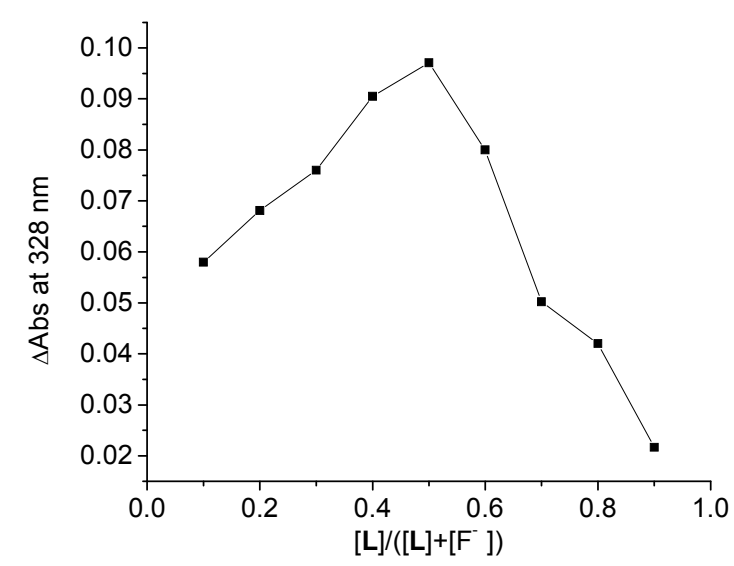

图 4 受体 $\mathbf{L}$ 与 $\mathrm{F}^{-}$作用的 Job 曲线(总浓度为 $40 \mu \mathrm{mol} / \mathrm{L}$ )

Figure 4 Job's plot between $\mathbf{L}$ and $\mathrm{F}^{-}$with a total concentration of $40 \mu \mathrm{mol} / \mathrm{L}$

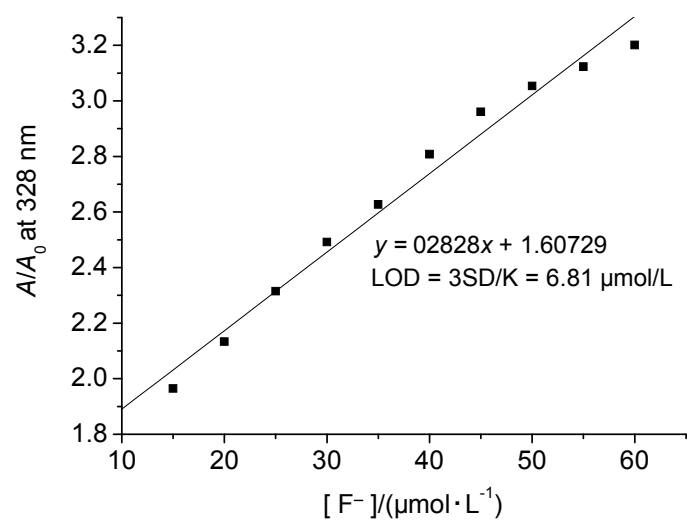

图 5 在 $\mathrm{DMSO}$ 中受体 $\mathbf{L}$ 对 $\mathrm{F}^{-}$的检测限

Figure 5 Determination of limit of detection of $\mathbf{L}$ for $\mathrm{F}^{-}$in DMSO

作用. 在含水 $10 \%$ 的 DMSO 中, $\mathbf{L}$ 能选择性识别 $\mathrm{F}^{-}$和 $\mathrm{AcO}^{-}$(图 6); 当测试体系中含水量提升至 $20 \%$ 时, $\mathbf{L}$ 对 $\mathrm{AcO}^{-}$表现出专一性识别作用.

\section{2 荧光光谱滴定}

考虑到咔唑类化合物具有良好的荧光发射性能, 受 体 $\mathbf{L}$ 对阴离子的识别作用接下来采用荧光光谱法加以 研究. 当激发波长为 $300 \mathrm{~nm}$ 时, 自由 $\mathbf{L}$ 在 $333 \mathrm{~nm}$ 处有 一明显的苂光发射峰(源于咔唑骨架). 如图 7 和图 8 所 示, 加入 $\mathrm{F}^{-} 、 \mathrm{AcO}^{-}$和 $\mathrm{H}_{2} \mathrm{PO}_{4}^{-}$后 $\mathbf{L}$ 的荧光出现明显猝 灭并伴随着肉眼可见的苂光颜色变化; 其它七种阴离子 的加入对 $\mathbf{L}$ 的苂光影响几乎可以忽略. 图 9 为受体 $\mathbf{L}$ 在 DMSO 中与 $\mathrm{F}^{-}$作用后的荧光光谱变化. 随着加入的 $\mathrm{F}^{-}$ 浓度不断增加, $\mathbf{L}$ 在 $333 \mathrm{~nm}$ 处的荧光逐渐减弱; 但其发 发射峰位置没有任何改变, 这表明 $\mathrm{F}^{-}$诱导的荧光猝灭 属于典型的光诱导电子转移(PET)机理 ${ }^{[14]}$. $\mathbf{L}$ 与 $\mathrm{F}^{-}$作用 后形成的超分子体系电子云密度增加, 这使得其向激发 态咔唑的电子转移变得更容易, 从而诱导了苂光猝灭;

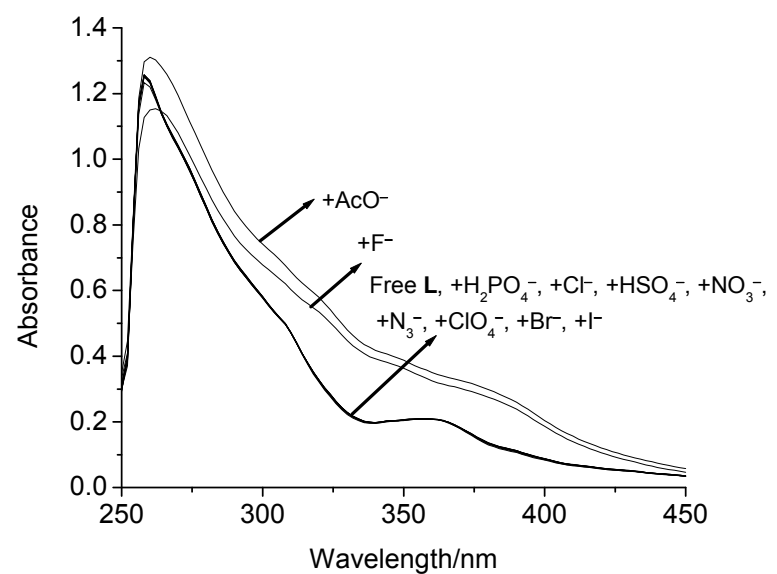

图 6 在含水 $10 \%$ 的 DMSO 中受体 $\mathbf{L}(20 \mu \mathrm{mol} / \mathrm{L})$ 与 16.5 equiv.不同阴离子作用后的 UV-Vis 光谱变化

Figure 6 UV-Vis spectral changes of receptor $\mathbf{L}(20 \mu \mathrm{mol} / \mathrm{L})$ upon addition of 16.5 equiv. of different anions in DMSO containing $10 \%(V / V) \mathrm{H}_{2} \mathrm{O}$

二者作用达到平衡后, $\mathbf{L}$ 苂光猝灭达 $34 \%$. 加入 $\mathrm{AcO}^{-}$或 $\mathrm{H}_{2} \mathrm{PO}_{4}^{-}$后, $\mathbf{L}$ 产生的荧光变化趋势与 $\mathrm{F}^{-}$类似. 由图 10 可知, $\mathbf{L}$ 在 $333 \mathrm{~nm}$ 处的荧光强度之比 $\left(I_{0} / I\right)$ 与 $\mathrm{F}^{-}$的浓度 $(10 \sim 80 \mu \mathrm{mol} / \mathrm{L})$ 呈现出良好的线性关系，这表明该浓度 范围可用于 $\mathrm{F}^{-}$的定量检测.

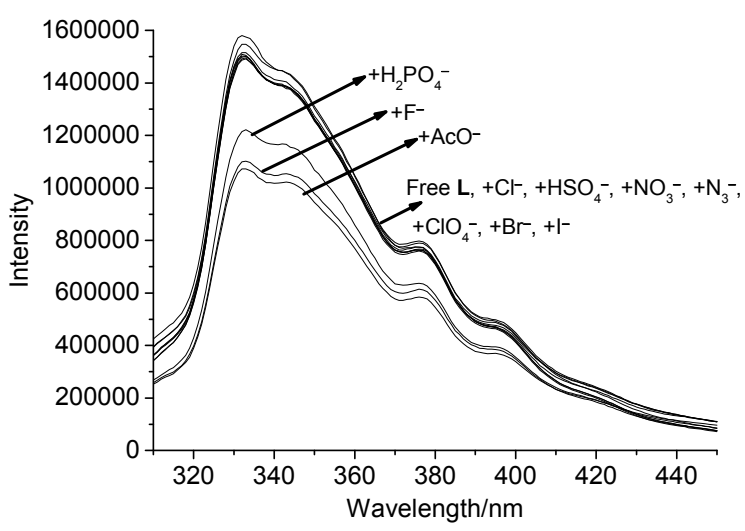

图 7 受体 $\mathbf{L}(20 \mu \mathrm{mol} / \mathrm{L})$ 在 $\mathrm{DMSO}$ 中与 3.5 equiv. 不同阴离子 作用后的荧光强度变化

Figure 7 Fluorescent changes of receptor $\mathbf{L}(20 \mu \mathrm{mol} / \mathrm{L})$ in DMSO after addition of 3.5 equiv. of various anions

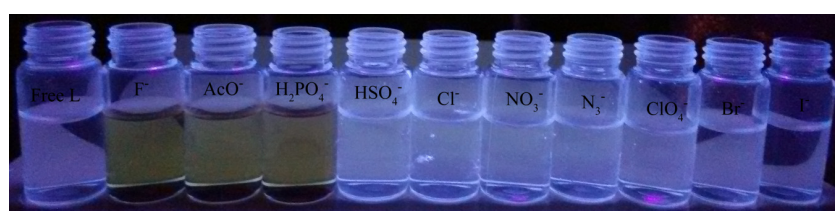

图 8 在受体 $\mathbf{L}(50 \mu \mathrm{mol} / \mathrm{L})$ 的 DMSO 溶液加入 5.0 equiv. 不同 阴离子后的苂光颜色变化

Figure 8 Fluorescent color changes of DMSO solution of receptor $\mathbf{L}(50 \mu \mathrm{mol} / \mathrm{L})$ upon addition of 5.0 equiv. of different anions under a UV-lamp $\left(\lambda_{\mathrm{ex}}=365 \mathrm{~nm}\right)$ 


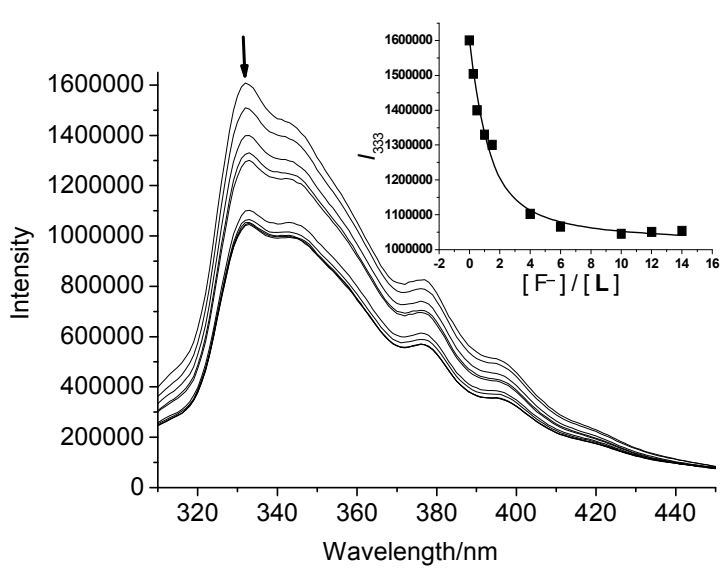

图 9 受体 $\mathbf{L}(20 \mu \mathrm{mol} / \mathrm{L})$ 在 $\mathrm{DMSO}$ 中与 $\mathrm{F}^{-}$作用后的荧光变化 Figure 9 Fluorescent changes of receptor $\mathbf{L}(20 \mu \mathrm{mol} / \mathrm{L})$ upon addition of $\mathrm{F}^{-}$in DMSO

The inset was the nonlinear curve fitting of the intensity at $333 \mathrm{~nm}$ against added $\mathrm{F}^{-}$using a $1: 1$ binding model

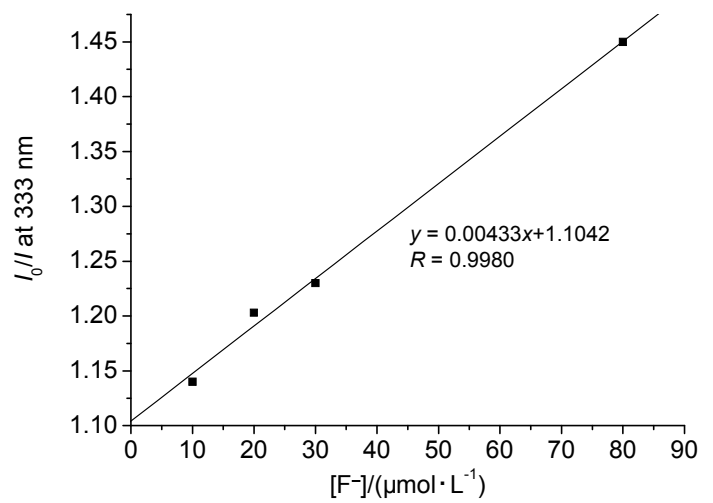

图 10 受体 $\mathbf{L}$ 在 $333 \mathrm{~nm}$ 处的 $I_{0} / I$ 与 $\left[\mathrm{F}^{-}\right]$之间的线性关系

Figure 10 A linear relationship between $I_{0} / I$ at $333 \mathrm{~nm}$ of $\mathbf{L}$ and the concentration of $\mathrm{F}^{-}$

在含水 $10 \%$ 的 $\mathrm{DMSO}$ 中, $\mathrm{F}^{-}$和 $\mathrm{AcO}^{-}$仍能诱导 $\mathbf{L}$ 发 生一定的苂光猝灭(图 11); 但当测试体系中含水量提升 至 $20 \%$ 时, $\mathbf{L}$ 则只对 $\mathrm{AcO}^{-}$表现出可测的苂光响应.

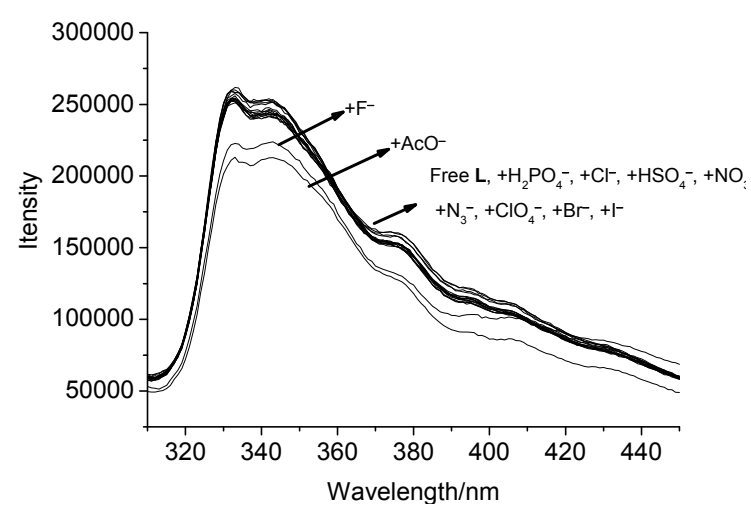

图 11 受体 $\mathbf{L}(20 \mu \mathrm{mol} / \mathrm{L})$ 在含水 $10 \%$ 的 DMSO 中与 15 equiv. 不同阴离子作用后的苂光变化

Figure 11 Fluorescent changes of receptor $\mathbf{L}(20 \mu \mathrm{mol} / \mathrm{L})$ in DMSO containing $10 \% \mathrm{H}_{2} \mathrm{O}$ after addition of 15 equiv. of various anions

\section{3 确定结合常数}

在超分子体系中, 若主客体之间形成化学计量比 为 $1: 1$ 的配合物, 可通过以下方程进行非线性拟合求 出结合常数 ${ }^{[15]}$.

$$
\begin{aligned}
& X=X_{0}+\left(X_{\lim }-X_{0}\right) / 2 C_{0}\left\{\left(C_{\mathrm{H}}+C_{\mathrm{G}}+1 / K_{\mathrm{a}}\right)-\left[\left(C_{\mathrm{H}}+\right.\right.\right. \\
& \left.\left.\left.C_{\mathrm{G}}+1 / K_{\mathrm{a}}\right)^{2}-4 C_{\mathrm{H}} C_{\mathrm{G}}\right]^{1 / 2}\right\}
\end{aligned}
$$

其中, $C_{\mathrm{H}}$ 为受体浓度, $C_{\mathrm{G}}$ 为加入的阴离子浓度, $K_{\mathrm{a}}$ 为结 合常数, $X_{0}$ 和 $X$ 分别为自由受体和加入阴离子后受体的 吸光强度(荧光滴定时则代表荧光强度). 若非线性拟合 求出的相关系数 $(R)$ 大于 0.99 , 则证明受体与阴离子形

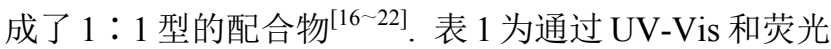
两种滴定法在不同体系中所求出的 $\mathbf{L}$ 与阴离子的结合 常数及相关系数. 从中可知, 在 $\mathrm{DMSO}$ 中受体 $\mathbf{L}$ 与 $\mathrm{AcO}^{-} 、 \mathrm{~F}^{-}$和 $\mathrm{H}_{2} \mathrm{PO}_{4}^{-}$均形成了 $1: 1$ 型配合物且对这三 种离子表现出强络合作用 $\left(K_{\mathrm{a}}>10^{4} \mathrm{~L} / \mathrm{mol}\right)$; 与我们 ${ }^{[12]}$ 最 近报道的咔唑磺酰朋受体相比, $\mathbf{L}$ 对上述三种阴离子的 $K_{\mathrm{a}}$ 增强都超过 15 倍 $\left(\mathrm{H}_{2} \mathrm{PO}_{4}^{-}\right.$达 25 倍, 基于 UV-Vis 拟 合结果). 两种不同光谱滴定法求出的亲和性顺序均为 $\mathrm{AcO}^{-}>\mathrm{F}^{-}>\mathrm{H}_{2} \mathrm{PO}_{4}^{-} \geqslant$其他阴离子, 这种顺序主要是由 相关阴离子的氢键接受能力所决定. 有趣的是, 在含水 $10 \%$ 的 $\mathrm{DMSO}$ 中 $\mathbf{L}$ 对 $\mathrm{F}^{-}$和 $\mathrm{AcO}^{-}$的结合常数仍大于 $10^{4}$ $\mathrm{L} / \mathrm{mol}$; 在含水 $20 \%$ 的 $\mathrm{DMSO}$ 中 $\mathrm{AcO}^{-}$与 $\mathbf{L}$ 也有中等强 度的亲和力 $\left(K_{\mathrm{a}}>10^{3} \mathrm{~L} / \mathrm{mol}\right)$.

\section{4 核磁滴定}

为探讨受体 $\mathbf{L}$ 与阴离子的识别机理，我们又通过核 磁滴定法做了进一步研究. 如图 12 所示, 在 DMSO- $d_{6}$ 中自由 $\mathbf{L}$ 在 $\delta 10.19 、 10.16 、 10.06$ 和 8.15 处出现四组 活泼 $\mathrm{NH}$ 信号 $\left(\mathrm{D}_{2} \mathrm{O}\right.$ 交换实验证实 $)$, 分别归属于咔唑 $\mathrm{NH}\left(\mathrm{H}_{1}\right)$ 、硫脲 $\mathrm{NH}\left(\mathrm{H}_{2}\right)$ 、硫脲 $\mathrm{NH}\left(\mathrm{H}_{3}\right)$ 和苯胺基 $\mathrm{NH}\left(\mathrm{H}_{4}\right)$ 质子. 加入 $0 \sim 0.9$ equiv. 的 $\mathrm{F}^{-}$后, $\mathrm{H}_{2}$ 信号峰缓慢向低场 移动并最终与 $\mathrm{H}_{1}$ 重合, $\mathrm{H}_{3}$ 和 $\mathrm{H}_{4}$ 的信号峰积分强度逐渐 减弱(无明显位移); 继续加入 $\mathrm{F}^{-}(0.9 \sim 6.5$ equiv.)后，合 并后的 $\mathrm{H}_{1}$ 和 $\mathrm{H}_{2}$ 新峰位置不变, $\mathrm{H}_{4}$ 信号峰强度继续减弱, $\mathrm{H}_{3}$ 信号峰消失. 在此过程中, 咔唑环上 $\mathrm{H}_{5}$ 和 $\mathrm{H}_{6}$ 分别向 高场移动了 $\delta 0.24$ 和 0.32 , 末端的苯环芳氢 $\left(\mathrm{H}_{7} 、 \mathrm{H}_{8}\right.$ 和 $\left.\mathrm{H}_{9}\right)$ 也出现了不同程度的高场移动 $(\delta \quad 0.06 \sim 0.11)$. 值得 一提的是, 在 6.5 equiv. 的 $\mathrm{F}^{-}$存在下, $\delta 16.10$ 处未出现归 属于 $\left[\mathrm{HF}_{2}^{-}\right]$的特征信号峰, 上述现象表明在 $0 \sim 6.5$ equiv. 的 $\mathrm{F}^{-}$浓度范围内 $\mathbf{L}$ 与 $\mathrm{F}^{-}$之间只建立了分子间氢键 作用而未发生 $\mathrm{NH}$ 去质子化. 综上所述, $\mathbf{L}$ 中 7 个 $\mathrm{NH}$ 单 元都参与了对 $\mathrm{F}^{-}$的氢键络合, 这影响了 $\mathbf{L}$ 的分子内电荷 转移(ICT)过程, 从而导致 $\mathbf{L}$ 发生了相应的 UV-Vis 和荧 光光谱变化. 根据以上实验结果, 提出了受体 $\mathbf{L}$ 与 $\mathrm{F}^{-}$的 可能结合模式(图 13). 加入 $\mathrm{AcO}^{-}$后, 受体 $\mathbf{L}$ 中 $\mathbf{N H}$ 和芳 
表 1 受体 $\mathbf{L}$ 在不同体系中与不同阴离子的结合常数 $\left(K_{\mathrm{a}}\right)$ 和相关系数 $(R)$

Table 1 Association constants $\left(K_{\mathrm{a}}\right)$ and correlation coefficients $(R)$ of receptor $\mathbf{L}$ with different anions in different solvents

\begin{tabular}{|c|c|c|c|c|c|c|}
\hline \multirow{2}{*}{ Anion $^{a}$} & \multicolumn{3}{|c|}{$K_{\mathrm{a}} /\left(10^{4} \mathrm{~L} \cdot \mathrm{mol}^{-1}\right), R(\mathrm{UV}-\mathrm{V} \text { is titration })^{b}$} & \multicolumn{3}{|c|}{$K_{\mathrm{a}} /\left(10^{4} \mathrm{~L} \cdot \mathrm{mol}^{-1}\right), R(\text { Fluorescence titration })^{b}$} \\
\hline & DMSO & $10 \% \mathrm{H}_{2} \mathrm{O}-\mathrm{DMSO}$ & $20 \% \mathrm{H}_{2} \mathrm{O}-\mathrm{DMSO}$ & DMSO & $10 \% \mathrm{H}_{2} \mathrm{O}-\mathrm{DMSO}$ & $20 \% \mathrm{H}_{2} \mathrm{O}-\mathrm{DMSO}$ \\
\hline $\mathrm{F}^{-}$ & $\begin{array}{c}(6.70 \pm 0.66) \\
0.9958\end{array}$ & $\begin{array}{c}(2.13 \pm 0.08) \\
0.9990\end{array}$ & $\begin{array}{c}\mathrm{ND}^{d}, \\
-\end{array}$ & $\begin{array}{c}(7.71 \pm 1.45) \\
0.9941\end{array}$ & $\begin{array}{c}(2.70 \pm 0.47) \\
0.9932\end{array}$ & $\begin{array}{c}\mathrm{ND}^{d} \\
-\end{array}$ \\
\hline $\mathrm{AcO}^{-}$ & $\begin{array}{c}(7.08 \pm 0.71) \\
0.9973\end{array}$ & $\begin{array}{c}(3.61 \pm 0.15) \\
0.9988\end{array}$ & $\begin{array}{c}(0.33 \pm 00.3) \\
0.9967\end{array}$ & $\begin{array}{c}12.0 \pm 1.6) \\
0.9943\end{array}$ & $\begin{array}{c}(4.18 \pm 0.51) \\
0.9975\end{array}$ & $\begin{array}{c}(0.65 \pm 0.01) \\
0.9897\end{array}$ \\
\hline $\mathrm{H}_{2} \mathrm{PO}_{4}^{-}$ & $\begin{array}{c}(4.35 \pm 0.16) \\
0.9987\end{array}$ & $\begin{array}{c}\mathrm{ND}^{d}, \\
-\end{array}$ & $\begin{array}{c}\mathrm{ND}^{d}, \\
-\end{array}$ & $\begin{array}{c}(7.07 \pm 0.98) \\
0.9920\end{array}$ & $\begin{array}{c}\mathrm{ND}^{d}, \\
-\end{array}$ & $\begin{array}{c}\mathrm{ND}^{d}, \\
-\end{array}$ \\
\hline Others ${ }^{c}$ & $\mathrm{ND}^{d}$ & $\mathrm{ND}^{d}$ & $\mathrm{ND}^{d}$ & $\mathrm{ND}^{d}$ & $\mathrm{ND}^{d}$ & $\mathrm{ND}^{d}$ \\
\hline
\end{tabular}

${ }^{a}$ All the anions were added as their tetrabutylammonium salts. ${ }^{b}$ All the values of $K_{\mathrm{a}}$ and $R$ were determined through the method of nonlinear curve fitting. ${ }^{c}$ Others included $\mathrm{Cl}^{-}, \mathrm{Br}^{-}, \mathrm{I}^{-}, \mathrm{NO}_{3}^{-}, \mathrm{N}_{3}^{-}, \mathrm{HSO}_{4}^{-}$and $\mathrm{ClO}_{4}^{-} \cdot{ }^{d}$ No or very slight spectral change was seen.

氢信号变化情况与 $\mathrm{F}^{-}$相似; 但加入 $\mathrm{H}_{2} \mathrm{PO}_{4}^{-}$后, 咔唑环 $\mathrm{H}_{6}$ 信号向低场移动了 $\delta 0.14$, 其余信号峰变化均与 $\mathrm{F}^{-}$类 似，这表明咔唑环 $\mathrm{H}_{6}$ 也通过跨空间效应参与了对 $\mathrm{H}_{2} \mathrm{PO}_{4}^{-}$的氢键络合.

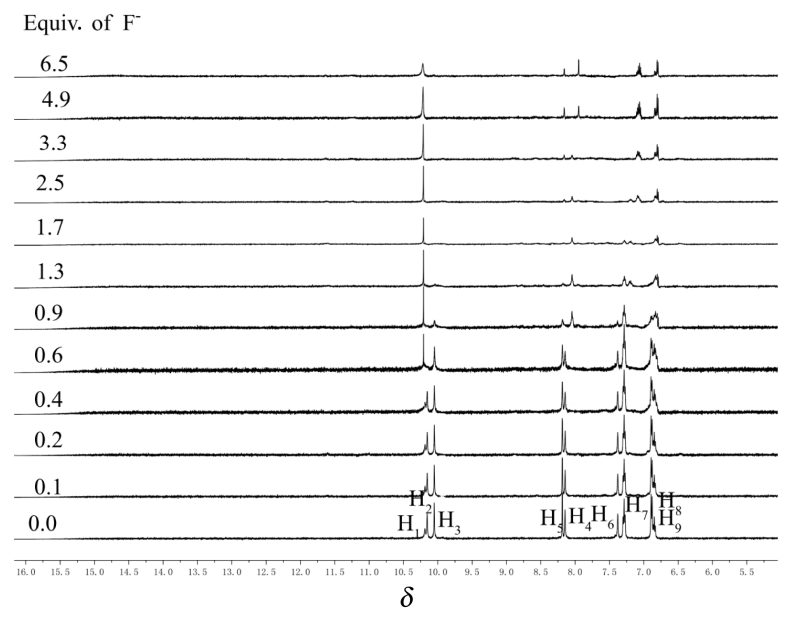

图 12 在 DMSO- $d_{6}$ 中受体 $\mathbf{L}(2.0 \mathrm{mmol} / \mathrm{L})$ 与 $\mathrm{F}^{-}$作用后的 ${ }^{1} \mathrm{H}$ NMR 变化图

Figure $12{ }^{1} \mathrm{H}$ NMR spectra of receptor $\mathbf{L}(2.0 \mathrm{mmol} / \mathrm{L})$ in DMSO- $d_{6}$ in the absence and presence of F

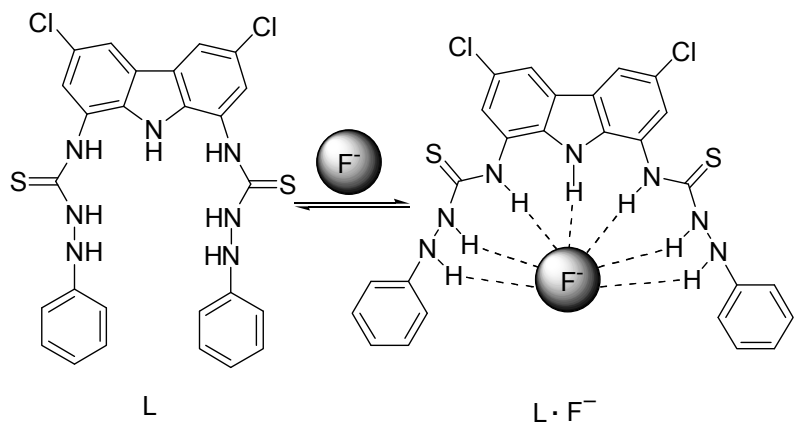

图 13 受体 $\mathbf{L}$ 与 $\mathrm{F}^{-}$在 DMSO 中的可能作用模式

Figure13 The possible binding model between receptor $\mathbf{L}$ and $\mathrm{F}^{-}$in DMSO

\section{2 结论}

我们将氨基硫脲作为阴离子识别单元引入到咔唑 骨架中设计合成了受体 $\mathbf{L}$, 通过 UV-Vis、裸眼观测、苂 光和核磁滴定研究了 $\mathbf{L}$ 在 DMSO 中对不同阴离子的识 别传感性质. 实验结果表明, $\mathbf{L}$ 能协同使用咔唑 9 位 $\mathrm{NH}$ 及苯胺基硫脲 $\mathrm{NH}$ 对 $\mathrm{F}^{-} 、 \mathrm{AcO}^{-}$和 $\mathrm{H}_{2} \mathrm{PO}_{4}^{-}$产生选择性 强络合作用 $\left(K_{\mathrm{a}}>10^{4} \mathrm{~L} / \mathrm{mol}\right)$, 识别过程伴随着肉眼可见 的溶液颜色变化和明显荧光猝灭，这表明 $\mathbf{L}$ 可同时作为 阴离子的比色和荧光传感器加以使用. 此外, 在含水 $10 \%$ 的 DMSO 中 $\mathbf{L}$ 能选择性识别 $\mathrm{F}^{-}$和 $\mathrm{AcO}^{-}$; 在含水 $20 \%$ 的 DMSO 中 $\mathbf{L}$ 则表现出对 $\mathrm{AcO}^{-}$的专一性识别.

\section{3 实验部分}

\section{1 仪器与试剂}

北京普析通用仪器有限公司的 TU-1900 型 UV-Vis 分光光度计; Horiba Scientific 公司的 Fluoromax-4 型荧 光光谱仪; JEOL-ECX $500 \mathrm{NMR}$ 核磁共振波谱仪(TMS 内标); Agilent LC/MSD Trap VL 型液质联用仪; Elementar Vario-III CHN 型元素分析仪; Shimadzu IR Prestige-21 型红外光谱仪; 北京泰克仪器有限公司的 XT-4 型显微熔点仪(温度计未校正). 干二氯甲烷、乙腈和三 乙胺由氢化钻回流除水制备, 其他试剂和溶剂均为市售 分析纯. 所用阴离子均为其四正丁基铵盐，1,8-二氨基3,6-二氯咔唑(2)的合成参照文献[23].

\section{2 实验方法}

\subsubsection{3,6-二氯-1,8-二异硫氧酸酯咔唑(1)的合成}

向溶有 $500 \mathrm{mg}(1.89 \mathrm{mmol}) 2$ 的 $100 \mathrm{~mL}$ 干二氯甲烷 和 $10 \mathrm{~mL}$ 干乙腈混合液中加入 $0.58 \mathrm{~mL}(7.55 \mathrm{mmol})$ 硫光 气后, 将上述体系冰浴冷却至 $0{ }^{\circ} \mathrm{C}$ 后缓慢滴加 $1.3 \mathrm{~mL}$ (9.43 mmol)干三乙胺, 滴加完毕后恢复至室温继续反应 $4 \mathrm{~h}$. 停止反应后向上述混合物中加入 $150 \mathrm{~mL}$ 饱和食盐 
水淬灭过量硫光气，分出有机层后用无水硫酸镁干燥， 浓缩后柱层析分离 [淋洗剂: $V$ (石油醚) : $V$ (乙酸乙酯 $)=$ 100：1]得淡黄色固体 $432 \mathrm{mg}$, 收率 65.5\%. m.p. 203 $206{ }^{\circ} \mathrm{C} ;{ }^{1} \mathrm{H}$ NMR (500 MHz, DMSO- $\left.d_{6}\right) \delta: 12.77$ (s, 1H), 8.38 (s, 2H), 7.82 (s, 2H); ${ }^{13} \mathrm{C}$ NMR (125 MHz, DMSO- $d_{6}$ ) $\delta: 136.2,134.0,126.1,125.0,124.6,121.2,115.8$; IR (KBr) v: 2922, 2152, 2027, 1483, 1303, 852, $578 \mathrm{~cm}^{-1}$.

3.2 .2 受体 $[3,6$-二氯-1,8-二(苯胺基硫脲)咔唑 $](\mathbf{L})$ 的 合成

在一单口烧瓶中加入中间体 $1100 \mathrm{mg}(0.28 \mathrm{mmol})$ 、 $10 \mathrm{~mL}$ 干乙腈和 $70 \mu \mathrm{L}(0.7 \mathrm{mmol})$ 苯肼, 氮气保护下加热 回流 $2 \mathrm{~h}$ 后, 停止反应后冷却至室温. 抽滤, 用乙腈和乙 醇洗涤滤渣, 干燥后得灰色固体 $88 \mathrm{mg}$, 收率 $55.6 \%$. m.p. $229 \sim 231{ }^{\circ} \mathrm{C}$; ${ }^{1} \mathrm{H}$ NMR (500 MHz, DMSO- $\left.d_{6}\right) \delta$ : $10.20\left(\mathrm{~s}, 1 \mathrm{H}, \mathrm{H}_{1}\right), 10.16\left(\mathrm{~s}, 2 \mathrm{H}, \mathrm{H}_{2}\right), 10.06\left(\mathrm{~s}, 2 \mathrm{H}, \mathrm{H}_{3}\right), 8.19$ $\left(\mathrm{s}, 2 \mathrm{H}, \mathrm{H}_{5}\right), 8.16$ (s, 2H, $\left.\mathrm{H}_{4}\right), 7.38\left(\mathrm{~s}, 2 \mathrm{H}, \mathrm{H}_{6}\right), 7.29$ (t, $J=$ $\left.7.6 \mathrm{~Hz}, 4 \mathrm{H}, \mathrm{H}_{7}\right), 6.89$ (d, $\left.J=7.9 \mathrm{~Hz}, 4 \mathrm{H}, \mathrm{H}_{8}\right), 6.85$ (t, $J=$ $\left.7.2 \mathrm{~Hz}, 2 \mathrm{H}, \mathrm{H}_{9}\right) ;{ }^{13} \mathrm{C}$ NMR (125 MHz, DMSO- $\left.d_{6}\right) \delta: 182.4$, $148.5,135.5,129.6,125.8,125.7,125.2,123.4,120.4$, 118.6, 113.9; IR (KBr) v: 3294, 3174, 1600, 1543, 1506, 1479, 1304, $750 \mathrm{~cm}^{-1}$; MS (ESI) $\mathrm{m} / z: 588.2[\mathrm{M}+\mathrm{Na}]^{+}$. Anal. calcd for $\mathrm{C}_{26} \mathrm{H}_{21} \mathrm{Cl}_{2} \mathrm{~N}_{7} \mathrm{~S}_{2}$ : C 55.12, H 3.74, N 17.31; found $\mathrm{C}$ 54.73, H 4.07, N 17.08.

辅助材料(Supporting Information) 本文所涉及到的 其它 UV-Vis、苂光和核磁滴定图谱. 这些材料可以免费 从本刊网站(http://sioc-journal.cn/)上下载.

\section{References}

[1] Gale, P. A. Chem. Soc. Rev. 2010, 39, 3746.

[2] Bondy, C. R.; Loeb, S. J. Coord. Chem. Rev. 2003, 240, 77.
[3] Beer, P. D.; Gale, P. A. Angew. Chem., Int. Ed. 2001, 40, 486.

[4] Zhou, Y.; Zhang, J. F.; Yoon, J. Chem. Rev. 2014, 114, 5511

[5] Pandurangan, K.; Kitchen, J. A.; Gunnlaugsson, T. Tetrahedron Lett. 2013, 54, 2770.

[6] Liu, B.; Tian, H. Chem. Lett. 2005, 34, 686.

[7] Gunnlaugsson, T.; Kruger, P. E.; Jensen, P.; Tierney, J.; Ali, H. D. P.; Hussey, G. M. J. Org. Chem. 2005, 70, 10875.

[8] Zhang, Y.; Xu, W.; Yao, H.; Wei, T. Chin. J. Chem. 2006, 24, 1406.

[9] Wei, W.; Zhang, Y.; Wei, T. Chin. J. Chem. 2008, 26, 1935.

[10] Cao, C.; Wei, T.; Wang, A.; Lin, Q.; Yao, H.; Zhang, Y. Prog. Chem. 2011, 23, 1069 ( in Chinese).

(曹成, 魏太保, 王爱霞, 林奇, 姚虹, 张有明, 化学进展, 2011, 23, 1069.)

[11] Liu, Y.; Zhang, F.; Zou, L.; Jian, J.; Bao, X. Chin. J. Org. Chem. 2013, 33, 2485 (in Chinese).

(刘勇, 张峰, 邹林波, 塞军友, 鲍小平, 有机化学, 2013, 33, 2485.)

[12] Zhang, F.; Tan, Z.; Yan, B.; Pan, D.; Bao, X. Chin. J. Org. Chem. 2014, 34, 2499 (in Chinese).

(张峰, 谭赞, 问柏任, 潘顶伍, 鲍小平, 有机化学, 2014, 34, 2499.)

[13] Chen, J.; Liu, C.; Zhang, J.; Ding, W.; Zhou, M.; Wu, F. Chem. Commun. 2013, 49, 10814.

[14] De Silva, A. P.; Gunaratne, H. Q. N.; Gunnlaugsson, T.; Huxley, A. J. M.; McCoy, C. P.; Rademacher, J. T.; Rice, T. E. Chem. Rev. 1997, 97, 1515.

[15] Valeur, B.; Pouget, J.; Bourson, J.; Kaschke, M.; Ernsting, N. P. J. Phys. Chem. 1992, 96, 6545.

[16] Bourson, J.; Pouget, J.; Valeur, B. J. Phys. Chem. 1993, 97, 4552.

[17] Schneider, H. J.; Kramer, R.; Simova, S.; Schneider, U. J. Am. Chem. Soc. 1988, 110, 6442.

[18] Wu, F.-Y.; Li, Z.; Wen, Z.-C.; Zhou, N.; Zhao, Y.-F.; Jiang, Y.-B. Org. Lett. 2002, 4, 3203.

[19] Liu, S.-Y.; Fang, L.; He, Y.-B.; Chan, W.-H.; Yung, K.-T.; Cheng, Y.-K.; Yang, R.-H. Org. Lett. 2005, 7, 5825.

[20] Bao, X.; Yu, J.; Zhou, Y. Sens. Actuators B 2009, 140, 467.

[21] Bao, X.; Zhou, Y. Sens. Actuators B 2010, 147, 434.

[22] Zhou, Y.-H.; Zheng, P.-C.; Bao, X.-P. Supramol. Chem. 2014, 26, 761.

[23] Chmielewski, M. J.; Charon, M.; Jurczak, J. Org. Lett. 2004, 6, 3501 .

(Qin, X.) 